\title{
Generating $\zeta$ with non-Abelian Vector Fields
}

\author{
Mindaugas Karčiauskas \\ CAFPE and Departamento de Física Teórica y del Cosmos, Universidad de Granada, \\ Granada-18071,Spain (mindaugas@ugr.es)
}

\begin{abstract}
In this paper the generation of the primordial curvature perturbation by vector fields of general non-Abelian groups is discussed. We show that non-Gaussianity of the perturbation is dominated by contributions from superhorizon evolution of fields. Also we find that non-Abelian vector fields of reasonably large groups can generate the total of the curvature perturbation without violating observational constraints on the angular modulation of the spectrum.
\end{abstract}

Keywords: Inflation, non-Gaussianity, statistical anisotropy

PACS: $98.80 .-\mathrm{k}, 98.80 . \mathrm{Cq}$

\section{INTRODUCTION}

Measuring statistical anisotropy alongside non-Gaussianity can give very important information about the physics of the very early Universe. It manifests itself as an angular modulation of the two-point and higher order correlation functions, for example, the quadrupole modulation of the spectrum [1]

$$
\mathcal{P}_{\zeta}(\mathbf{k})=\mathcal{P}_{\zeta}^{\text {iso }}(k)\left[1+g_{\zeta}(\hat{\mathbf{n}} \cdot \hat{\mathbf{k}})\right],
$$

where $\hat{\mathbf{n}}$ is the preferred direction. Currently two observational bounds exist in the literature on primordial $g_{\zeta}:\left|g_{\zeta}\right|<0.3$ [2] and $\left|g_{\zeta}\right|<0.07$ [3]. The Planck satellite should better this bound with predicted accuracy of $0.01(2 \sigma)$ [4]. But even if $g_{\zeta}$ is found to be negligible, the angular modulation can still be dominant in higher order correlators [5].

Constraints on statistical anisotropy are especially useful in determining possible effects of vector fields in the very early Universe. Their non-negligible role could be expected as vector fields are ubiquitous in particle physics models. Apart from other effects, they could have generated the total or part of $\zeta[6]$ or influenced the dynamics of the inflaton [7]. From a theoretical side, scenarios with vector fields open a new window for the inflationary model building [8].

In this work we show that non-Abelian vector fields of reasonably large groups can also generate $\zeta$ without violating observational bounds on $g_{\zeta}$. Moreover, its statistical properties are dominated by perturbations of classical fields [9].

\section{THE LAGRANGIAN}

We consider a Lagrangian of massless, non-Abelian vector fields with time dependent kinetic function

$$
\mathcal{L}=-\frac{1}{4} f(t) F_{\mu \nu}^{a} F_{a}^{\mu \nu}
$$


where the field strength tensor $F_{\mu \nu}^{a}$ is given by

$$
F_{\mu \nu}^{a}=\partial_{\mu} A_{\nu}^{a}-\partial_{\nu} A_{\mu}^{a}+g_{\mathrm{c}} \mathrm{f}^{a b c} A_{\mu}^{b} A_{\nu}^{c} .
$$

and $\mathrm{f}^{a b c}$ are the Lie algebra structure constants of any non-Abelian group. We assume that the gauge kinetic function $f(t)$ is modulated by some of the dynamical degrees of freedom of the full theory, which can be the inflaton itself [8]. But in this work we do not need to specify the origin of this modulation. Instead, we assume that $f(t)$ is not modulated by the inflaton and that vector fields make a negligible contribution to the total energy budget of the Universe.

Note, that vector fields $A_{\mu}^{a}$ in Eq. (3) are defined with respect to the comoving coordinates [10]. Spatial components of physical vector fields are given by $W_{i}^{a}=$ $\sqrt{f} A_{i}^{a} / a$, where we also canonically normalised the field. Also note, that the selfcoupling coefficient $g_{\mathrm{c}} / \sqrt{f(t)}$ of canonically normalised fields is time-dependent.

In Ref. [5] it was shown that both degrees of freedom of a massless Abelian vector field acquire a flat perturbation spectrum $\mathcal{P}_{\delta W}=(H / 2 \pi)^{2}$ if $f \propto a^{-4}$. In this case $f \gg 1$ during inflation and self-couplings are suppressed, which also results in flat perturbation spectra of $W_{\mu}^{a}$ fields.

\section{STATISTICALLY ANISOTROPIC PERTURBATIONS}

Self-interactions of non-Abelian vector fields render their perturbations non-Gaussian. We calculate the non-Gaussianity using the "in-in formalism" [11]. Limiting ourselves only to the tree level bispectrum it is enough to consider two contributions to the unitary

evolution of states. Writing the interaction Hamiltonian as $\hat{H}_{\text {int }}=\hat{H}_{\text {int }}^{(3)}+\hat{H}_{\text {int }}^{(4)}$ the second of these contribution is

$$
\hat{H}_{\mathrm{int}}^{(4)}=a^{4}(\tau) \int \mathrm{d}^{3} \times \frac{1}{2} \frac{g_{\mathrm{c}}^{2}}{f}\left(\mathrm{f}^{a b c} \mathrm{f}^{a d e}+\mathrm{f}^{a d c} \mathrm{f}^{a b e}\right) W_{i}^{b} \delta \hat{W}_{j}^{c} \delta \hat{W}_{i}^{d} \delta \hat{W}_{j}^{e},
$$

where $\delta$ denotes the perturbation of the field. The first contribution is proportional to the gradient and thus is suppressed on superhorizon scales.

For the field operators we impose Bunch-Davies initial conditions. Integrating the expectation value of the three point correlator we find the three-point correlation function at the end of inflation as

$$
g_{3}\left(\mathbf{k}_{1}, \mathbf{k}_{2}, \mathbf{k}_{3}\right)=-(2 \pi)^{3} \delta\left(\mathbf{k}_{1}+\mathbf{k}_{2}+\mathbf{k}_{3}\right) \frac{2 H^{6}}{\prod_{i}^{3} 2 k_{i}^{3}} \mathcal{T}_{l m n}^{f g h}\left(\hat{\mathbf{k}}_{1}, \hat{\mathbf{k}}_{2}, \hat{\mathbf{k}}_{3}\right) I\left(k_{1}, k_{2}, k_{3}\right)
$$

where group indices on $g_{3}$ are suppressed for brevity. First, note that the three point correlator is anisotropic:

$$
\begin{aligned}
\mathcal{T}_{l m n}^{f g h}\left(\hat{\mathbf{k}}_{1}, \hat{\mathbf{k}}_{2}, \hat{\mathbf{k}}_{3}\right) \equiv & W_{m}^{b} T_{l j}\left(\hat{\mathbf{k}}_{1}\right) T_{n j}\left(\hat{\mathbf{k}}_{3}\right)\left(\mathrm{f}^{a b h} \mathrm{f}^{a g f}+\mathrm{f}^{a g h} \mathrm{f}^{a b f}\right)+ \\
& +W_{l}^{b} T_{m j}\left(\hat{\mathbf{k}}_{2}\right) T_{n j}\left(\hat{\mathbf{k}}_{3}\right)\left(\mathrm{f}^{a b g} \mathrm{f}^{a f h}+\mathrm{f}^{a f g} \mathrm{f}^{a b h}\right)+ \\
& +W_{n}^{b} T_{l j}\left(\hat{\mathbf{k}}_{1}\right) T_{m j}\left(\hat{\mathbf{k}}_{2}\right)\left(\mathrm{f}^{a b g} \mathrm{f}^{a h f}+\mathrm{f}^{a h g} \mathrm{f}^{a b f}\right)
\end{aligned}
$$


where tensors $T_{i j}(\hat{\mathbf{k}}) \equiv \delta_{i j}-\hat{k}_{i} \hat{k}_{j}$ depend on the direction of wave vectors. The amplitude $I$ in Eq. (5) is given by

$$
\begin{aligned}
I= & \frac{g_{\mathrm{c}}^{2}}{f_{0}} \frac{k_{\mathrm{t}}^{7} H^{-8}}{4 !}\left[6 \mathrm{e}^{4 N_{k}}\left(\frac{1}{3}-K_{1}+K_{2}\right)+2 \mathrm{e}^{2 N_{k}}\left(K_{1}-3 K_{2}-\frac{1}{5}\right)-\right. \\
& \left.-\left(\gamma+N_{k}\right)\left(\frac{1}{5} K_{1}-K_{2}-\frac{1}{35}\right)+\frac{1}{300}\left(625 K_{2}-137 K_{1}+\frac{1019}{49}\right)\right],
\end{aligned}
$$

where $f_{0}$ is the initial value of $f, \gamma \approx 0.577$ is the Euler-Macheroni constant and $k_{\mathrm{t}} \equiv$ $k_{1}+k_{2}+k_{3}, K_{1} \equiv \sum_{i>j}^{3} k_{i} k_{j} / k_{\mathrm{t}}^{2}, K_{2} \equiv \prod_{i}^{3} k_{i} / k_{\mathrm{t}}^{3}$. The parameter $N_{k} \equiv-\ln \left(k_{\mathrm{t}} / a_{\text {end }} H\right)$ is the number of e-foldings from the horizon crossing of $k_{\mathrm{t}}$ to the end of inflation. For cosmological scales $N_{k} \sim 60$ and the first term in the above expression is the dominant one, i.e. the bispectrum is dominated by the classical contribution. Indeed, in Ref. [9] it was explicitly shown that solutions of classical field equations give the same result as $g_{3}$ in Eq. (5) with the dominant term in Eq. (7) given by

$$
I \approx \frac{g_{\mathrm{c}}^{2}}{f_{\mathrm{e}}} \frac{4 \pi^{4}}{12 H^{2}} \mathcal{P}_{\delta W}^{2}
$$

where $f_{\mathrm{e}}$ is evaluated at the end of inflation.

We calculate the curvature perturbation $\zeta$ using the $\delta N$ formula and assume that only vector field contributions are considerable. Since all vector fields satisfy the same equation of motion and structure constants $\mathrm{f}^{a b c}$ are of the same order, it is reasonable to assume that homogeneous values of all vector fields are of the same order of magnitude and their orientations in space are random. If this is the case, the power spectrum of $\zeta$ becomes

$$
\mathcal{P}_{\zeta}(\mathbf{k})=\mathcal{N} \mathcal{P}_{\delta W} N_{W}^{2}\left[1-\frac{1}{\mathcal{N}} \sum_{a}^{\mathcal{N}}\left(\hat{\mathbf{W}}^{a} \cdot \hat{\mathbf{k}}\right)^{2}\right],
$$

where hats denote unit vectors, $N_{W} \approx\left|\partial N / \partial W_{i}^{a}\right|$ for all $a$ and $N$ is the number of efoldings of unperturbed expansion. $\mathcal{N}$ in the above is the number of vector fields. As we can see, the angular modulation of $\mathcal{P}_{\zeta}$ is suppressed by $\mathcal{N}$. Thus vector fields of a large enough non-Abelian group can generate the curvature perturbation without violating observational bounds on $g_{\zeta}$. In view of the two bounds on $g_{\zeta}, \mathcal{N} \geq 4$ or 15 if $\left|g_{\zeta}\right|<0.29$ or 0.07 respectively.

\section{GENERATING ANISOTROPIC $\zeta$ AT THE END OF INFLATION}

The end-of-inflation scenario with a vector field was first studied in Ref. [12]. Here we employ this scenario in a toy example, motivated by particle physics models. Consider a Lagrangian which is invariant under transformations of some non-Abelian symmetry group $G$

$$
\mathcal{L}=\frac{1}{2} \partial_{\mu} \varphi \partial^{\mu} \varphi-\frac{1}{4} f F_{a}^{\mu \nu} F_{\mu \nu}^{a}+\frac{1}{2} \operatorname{Tr}\left[\left(D_{\mu} \Phi\right)^{\dagger} D^{\mu} \Phi\right]-V(\varphi, \Phi)
$$


where $\operatorname{Tr}[\ldots]$ stands for trace, $\varphi$ is the inflaton field and $F_{\mu \nu}^{a}$ is the field strength tensor defined in Eq. (3). $\Phi$ in the above is the Higgs field corresponding to a non-trivial representation of $G$ and the covariant derivative is $D_{\mu}=\partial_{\mu}+i \lambda_{A} \mathbf{T}^{a} A_{\mu}^{a}$, where $\lambda_{A}$ is the gauge coupling constant and $\mathbf{T}^{a}$ are generators of the symmetry group $G$. Rewriting the Higgs field as $\Phi \equiv \phi \mathbf{l}$, where $\mathbf{l}$ defines the direction of symmetry breaking in the field space with $\operatorname{Tr}\left[\mathbf{l}^{\dagger} \mathbf{l}\right]=1$, the potential $V(\varphi, \Phi)$ takes the form

$$
V(\varphi, \phi)=\frac{1}{4} \lambda\left(\phi^{2}-M^{2}\right)^{2}+\frac{1}{2} \kappa^{2} \varphi^{2} \phi^{2}+V(\varphi) .
$$

One can easily recognize this as the potential of hybrid inflation in which $\phi$ is stabilised at the origin during inflation. But in contrast to the standard hybrid inflation, in this example $\zeta$ is generated by gauge fields at the end of inflation. To see this, note from Eqs. (10) and (11) that the effective mass squared of the Higgs field $\phi$ is

$$
m_{\text {eff }}^{2}(\mathbf{x})=\kappa^{2} \varphi^{2}-\lambda M^{2}-\lambda_{A}^{2} A_{\mu}^{a} A_{b}^{\mu} \mathbf{1}^{\dagger} \mathbf{T}^{a} \mathbf{T}^{b} \mathbf{l} .
$$

In this scenario the curvature perturbation power spectrum in Eq. (9) becomes

$$
\mathcal{P}_{\zeta}(\hat{\mathbf{k}}) \approx \lambda_{A}^{4} \mathcal{N} \mathcal{P}_{\delta W}(C W)^{2}\left[1-\frac{1}{\mathcal{N}} \sum_{\bar{a}}\left(\hat{\mathbf{W}}^{\bar{a}} \cdot \hat{\mathbf{k}}\right)^{2}\right],
$$

where we used $W \approx\left|\mathbf{W}^{\bar{a}}\right|$ for all $\bar{a}$ and indices with overbar run only over generators broken at the end of inflation. Also in the above $C \equiv N_{\mathrm{e}} / \kappa^{2} f_{\mathrm{e}} \varphi_{\mathrm{c}}$, where $\varphi_{\mathrm{c}}$ is the critical value of the inflaton and $N_{\mathrm{e}} \equiv \partial N / \partial \varphi_{\mathrm{c}}$. Note that $\mathcal{N}$ in Eq. (13) is the number of massive vector fields.

As was noted before, the bound on $\mathcal{N}$ is $\mathcal{N} \geq 4$ or 15 . Assuming $G$ to be a special unitary group $S U(N)$ which at the phase transition is broken to $S U(N-1)$, the symmetry breaking results in $\mathcal{N}=2 N-1$ massive gauge fields. Thus, a weaker bound on $\mathcal{N}$ is satisfied with the $S U(3)$ group while the stronger bound requires $S U(8)$. Therefore $\zeta$ can be generated by gauge fields of reasonably large groups.

Acknowledgments MK is supported by CPAN CSD2007-00042 and MICINN (FIS2010-17395) grants.

\section{REFERENCES}

1. L. Ackerman, S. M. Carroll, and M. B. Wise, Phys. Rev. D75, 083502 (2007).

2. N. E. Groeneboom, L. Ackerman, I. K. Wehus, and H. K. Eriksen, Astrophys.J. 722, 452-459 (2010).

3. D. Hanson, A. Lewis, and A. Challinor, Phys. Rev. D81, 103003 (2010).

4. Y.-Z. Ma, G. Efstathiou, and A. Challinor, Phys.Rev. D83, 083005 (2011).

5. K. Dimopoulos, M. Karčiauskas, and J. M. Wagstaff, Phys. Rev. D81, 023522 (2010).

6. K. Dimopoulos, Phys.Rev. D74, 083502 (2006).

7. S. Kanno, M. Kimura, J. Soda, and S. Yokoyama, JCAP 0808, 034 (2008).

8. K. Dimopoulos, G. Lazarides, and J. M. Wagstaff, arXiv: 1111.1929 [astro-ph.CO] .

9. M. Karčiauskas, JCAP 1201, 014 (2012).

10. K. Dimopoulos, M. Karčiauskas, D. H. Lyth, and Y. Rodriguez, JCAP 05, 013 (2009).

11. J. M. Maldacena, JHEP 0305, 013 (2003).

12. S. Yokoyama, and J. Soda, JCAP 08, 005 (2008). 